\section{Kidney Blood Pressure Research}

\title{
Resveratrol Ameliorates Contrast Induced Nephropathy Through the Activation of SIRT1-PGC-1 $\alpha$-Foxo1 Signaling in Mice
}

\author{
Yu Ah Hong ${ }^{a}$ So Yeon Bae ${ }^{b}$ Shin Young Ahn ${ }^{b}$ Jieun Kim ${ }^{b}$ Young Joo Kwon \\ Woon Yong Jung ${ }^{c}$ Gang Jee Ko \\ aDepartment of Internal Medicine, College of Medicine, The Catholic University of Korea, Seoul, \\ bDepartment of Internal Medicine, Korea University College of Medicine, Seoul, 'Department of \\ Pathology, International St. Mary`s Hospital, Incheon, Republic of Korea
}

\section{Key Words}

Contrast media $\bullet$ Acute kidney injury $\bullet$ Resveratrol $\bullet$ Oxidative stress $\bullet$ Apoptosis $\bullet$ SIRT1

\begin{abstract}
Background/Aims: SIRT1 activation promotes the resistance of renal tubular cells to oxidative stress, and resveratrol is known as a SIRT1 activator. Methods: Resveratrol was injected intraperitoneally with iohexol for 24 hours. NRK-52E cells were pretreated with resveratrol for 24 hours and then exposed to iohexol for 3 hours. Renal function was measured by serum creatinine and cell survival was assessed by MTT assay. We investigated whether resveratrol attenuates oxidative stress and apoptosis in contrast-induced nephropathy (CIN). Results: Serum creatinine and tubular injury increased significantly after iohexol treatment, and resveratrol co-treatment attenuated the renal injury. Cell survival decreased after iohexol exposure and resveratrol reduced cell death induced by iohexol. Resveratrol was accompanied with the activation of SIRT1 and PGC-1 $\alpha$ and dephosphorylation of FoxO1 in mice with CIN. SIRT1 and PGC-1 $\alpha$ expression were decreased by iohexol, and increased significantly in resveratrol-pretreated cells. These processes resulted in reduction of oxidative stress and apoptosis both in vivo and in vitro experiments. Resveratrol decreased inflammatory cell infiltration induced by iohexol in mice with CIN. SIRT1 inhibition using siRNA in tubular cells accentuated the decrease of cell viability by iohexol. Conclusion: Resveratrol attenuated CIN by modulating renal oxidative stress and apoptosis through activation of SIRT1-PGC-1 $\alpha$ FoxO1 signaling.

\section{Introduction}

Contrast-induced nephropathy (CIN) is one of common causes of acute kidney injury among inpatients following the intravascular administration of contrast media (CM), such as 


\section{Kidney Blood Pressure Research}

computed tomography, angiography and interventions [1]. CIN remains a substantial medical issue because it is associated with delayed duration of hospitalization, the potential need for dialysis, and decreased patient's survival [2]. In spite of numerous attempts to reduce the incidence of CIN, such as use of prophylactic antioxidants and development of lower osmolar contrast agents, which are supposed to be less nephrotoxic, preventive measures with proven efficacy have not been confirmed, except rigorous hydration [3-5]. Although the definite pathogenesis of CIN has not been fully understood, it is mainly believed to be related to hypoxia due to $\mathrm{CM}$-induced renal vasoconstriction and decrease in oxygen supply, with a subsequent oxidative stress and cellular apoptosis related to enhanced production of reactive oxygen species (ROS), leading to renal tubular toxicity [6-8]. However, the detailed molecular mechanism underlying this oxidative stress and cellular apoptosis remains poorly understood.

Sirtuin 1 (SIRT1) is a member of the highly preserved nicotinamide adenine dinucleotide $\left(\mathrm{NAD}^{+}\right)$-dependent class III histone deacetylases and an extremely sensitive energy sensor [9]. SIRT1 has roles in diverse processes including energy metabolism, response to stress, hormone reactions, and cell apoptosis. SIRT1 deacetylates histones and a number of transcription factors and cofactors, such as p53 [10], forkhead box (FoxOs) proteins [11], peroxisome proliferator-activated receptor- $\gamma$ co-activator $1 \alpha$ (PGC-1 $\alpha)$ [12], and nuclear factor-kB [13], and affects crucial pathways involved in the response of cellular stress. Activation of SIRT1 promotes the resistance of renal tubular cells to oxidative stress [14]. Previous studies also showed that specific overexpression of SIRT1 attenuates cisplatininduced nephropathy, renal ischemic/reperfusion injury, and diabetic nephropathy [15-17].

PGC- $1 \alpha$ is a transcription coactivator that regulates cellular energy metabolism and is mainly expressed in tissue with abundant oxidative capacity, such as brown adipose tissues, heart, brain, skeletal muscle and kidney. PGC-1 $\alpha$ attenuates oxidative stress by increasing expression of Mn superoxide dismutase (SOD2), a major mitochondrial antioxidant enzyme, and suppressing ROS $[18,19]$. Thus, activating of SIRT1 and PGC- $1 \alpha$ may have a beneficial renoprotective effect on reducing intracellular oxidative stress and apoptosis. Resveratrol (RSV), which is a natural polyphenolic compound, has been known to be a SIRT1 activator and shown a protective effect for various diseases through antioxidant and anti-apoptotic effects [20]. We hypothesized that resveratrol would reduce oxidative stress and tubular apoptosis and prevent CIN by activating the SIRT1-PGC- $1 \alpha$ signaling pathway.

\section{Materials and Methods}

\section{Animals and experimental design}

Male C57BL/6J mice (aged 7-10-weeks) were purchased from Orient-Bio (Gyeonggi-do, Korea). All mice were housed in a facility with an alternating 12 -hour light/dark cycle at $20^{\circ} \mathrm{C}, 60 \%$ relative humidity, and acclimated for 7 days before the experiments. The mice were fed a commercial chow and allowed free access to water until 12 hours before the experiments. The mice were assigned randomly to four groups: (1) saline-treated control group (Cont; $n=6$ ), (2) resveratrol-treated group (Res; $n=6$ ), (3) CM-treated group (CM; $n=14)$, and (4) CM plus resveratrol-treated group ( $\mathrm{CM}+$ Res; $\mathrm{n}=14$ ). Iohexol (Omnipaque; $350 \mathrm{mg}$ iodine/mL, 5 g iodine/kg; GE Healthcare, Princeton, NJ, USA) was used as the iodinated contrast media in the present study. A model of CIN in mice, combining an injection of a nitric oxide synthase inhibitor $\left(\mathrm{N}^{\mathrm{G}}-\right.$ nitro-L-arginine methyl ester, $10 \mathrm{mg} / \mathrm{kg}$; Sigma-Aldrich, St. Louis, MO, USA) and a prostaglandin synthesis inhibitor (indomethacin, $10 \mathrm{mg} / \mathrm{kg}$; Sigma-Aldrich), was described previously [21]. All groups received $\mathrm{N}^{\mathrm{G}}$-nitro-L-arginine methyl ester and indomethacin $30 \mathrm{~min}$ prior to the saline and iohexol injections, and all drugs were injected intraperitoneally. Noteworthy, iohexol appearance in the urinary collecting system 1-2 hours following its intraperitoneal administration was confirmed by repeated imaging. To determine whether SIRT1 activators protect against CIN in mice, resveratrol (30 mg/kg; Sigma-Aldrich), a natural plant polyphenol known as a potent drug increasing sirtuin activity, was simultaneously injected with saline or iohexol via an intraperitoneal route. The most effective dose of resveratrol to increase SIRT1 


\section{Kidney Blood Pressure Research}

Hong et al.: Renoprotective Effects of Resveratrol in CIN

activity was selected based on our preliminary experiment. The experiment protocol for this study was approved by the Institutional Animal Care and Use Committee of Korea University College of Medicine (KUIACUC-20130423-5), and the experiments were performed in accordance with animal care guidelines of our institution. All mice were anesthetized with an injection of ketamine via an intraperitoneal route and killed at 24 hours after drug administration.

\section{Quantification of functional and morphological kidney injury}

Blood samples were drawn from the inferior vena cava, and both kidneys were harvested after full exsanguination. Serum creatinine concentrations were measured using the DetectX creatinine serum detection kit (Arbor Assays; Ann Arbor, MI, USA). The kidneys were retrieved, washed with saline, fixed in $4 \%$ paraformaldehyde, and embedded in paraffin. The renal sections were stained with periodic acidSchiff (PAS). Renal tubular injury was scored by a renal pathologist in a blinded manner to the experimental groups ( $\times 400$ magnification). A grading scale of $0-4$, according to the proportion of injured tubular area [22], was used for the histological measurement of contrast-induced kidney injury (grade $0=0 \%$, grade $1=$ $0-25 \%$, grade $2=25-50 \%$, grade $3=50-75 \%$, and grade $4=75-100 \%$ ).

\section{Cell culture}

The NRK-52E rodent renal tubular cell line (American Type Culture Collection, Manassas, VA, USA) was cultured in Dulbecco's Modified Eagle's Medium (GIBCO, Life Technologies, Seoul, Korea) and the medium was replaced every 2 to 3 days. An in vitro model of CIN was used as described previously [21, 23]. Cells were exposed to iohexol $(100 \mathrm{mg} / \mathrm{mL})$ for 3 hours. Equal volumes of saline were used as the control. To evaluate the effect of activating SIRT1, the cells were pretreated with resveratrol $(0,10$, and $50 \mu \mathrm{mol})$ for 24 hours before the iohexol treatment.

\section{MTT cytotoxic assay}

To evaluate the degree of cytotoxicity induced by iohexol after the co-treatment with or without resveratrol, cell viability was measured by the 3-[4, 5-dimethyl(thiazol-2- yl)-3, 5-dipheryl] tetradium bromide (MTT) cytotoxic assay. The MTT assay was used for measuring mitochondrial dehydrogenase activity, as a component of the tricarboxylic acid cycle and a marker of energy production. Cells were incubated with $1 \mathrm{mg} / \mathrm{mL}$ MTT (in sterile PBS) for 1 hour at $37^{\circ} \mathrm{C}$ and dissolved in dimethyl sulfoxide. Optical density was quantified at $570 \mathrm{~nm}$ using a Beckman spectrophotometer (Brea, CA, USA).

\section{Immunoblot analysis}

Proteins were extracted from kidney tissues, and their concentration was determined using Bradford solution (Bio-Rad, Hercules, CA, USA). A $100 \mu \mathrm{g}$ of protein was electrophoresed on SDS-polyacrylamide gels under denaturing conditions, and electroblotted to a polyvinylidene fluoride membrane (Millipore, Bedford, MA, USA). The membranes were incubated with 5\% non-fat dry milk in Tris-buffered saline Tween-20 (TBST) buffer for 1 hour at room temperature. The membrane was hybridized with polyclonal antibodies against SIRT1 (1:1, 000; Cell Signaling Technology, Danvers, MA, USA), PGC-1 $\alpha$ (1:1, 000; Novus Biologicals, Littleton, CO, USA), total-FoxO3a (1:1, 000; Cell Signaling Technology), phospho-Ser ${ }^{253}$ FoxO3a (1:1, 000; Cell Signaling Technology), total-Fox01 (1:1, 000; Cell Signaling Technology), phospho-Ser ${ }^{256}$ Fox01 (1:1000; Cell Signaling Technology), Mn superoxide dismutase (SOD2, 1:1, 000; Santa Cruz Biotechnology), and cleaved caspase-3 $\left(1: 1,000\right.$; Cell Signaling Technology) overnight at $4^{\circ} \mathrm{C}$. The membranes were reacted with horseradish peroxidase-conjugated secondary antibody (1:2, 000; Vector Laboratories) for 90 min. Signals were visualized by chemiluminescent detection according to the manufacturer's protocol (Amersham Pharmacia Biotech, London, UK), and signal density was measured using ImageJ software (http://rsbweb.nih. gov/ij). Equal amounts of protein loading were confirmed by $\beta$-actin $(1: 20,000$; Santa Cruz Biotechnology) or $\alpha$-tubulin (1:1, 000; Cell Signaling Technology).

\section{Immunohistochemical staining and TUNEL assay}

After deparaffinization, the kidney sections were hydrated in a graded ethanol series, treated with $0.1 \%$ trypsin (Zymed, San Francisco, CA, USA) in $0.3 \% \mathrm{H}_{2} \mathrm{O}_{2}$, and incubated with blocking serum (Vector, Peterborough, UK) to prevent any nonspecific reactions. Then, the slides were incubated overnight at $4^{\circ} \mathrm{C}$ 


\section{Kidney Blood Pressure Research}

with F4/80 antibody (1:100, AbD Serotec, Kidlington, UK), and reacted with a biotin-conjugated secondary antibody. For colorization, The avidin-biotin horseradish peroxidase complex and a 3, 3-diamninobenzidine substrate solution (Vector Laboratories, Burlingame, CA, USA) were applied to color the slides at room temperature, followed by counterstaining with hematoxylin (Sigma-Aldrich). The negative control was stained under identical conditions with rabbit serum substituting for the primary antibody. All sections were examined in a blinded manner using a light microscope (Olympus BX-50; Olympus Optical, Tokyo, Japan). Eight to ten high power fields (HPFs) were captured $(\times 400$ magnification), and the mean number of F4/80 positively stained cells was calculated for quantification.

Apoptotic cells were counted in the kidney on paraffinembedded kidney tissue sections using ApopTag Plus (Intergen, Purchase, NY, USA) following the manufacturer's protocol. Eight to ten HPFs were also captured ( $\times 400$ magnification), and terminal deoxynucleotidyl transferase-mediated dUTP nickend labeling (TUNEL)-positive cells were counted in the outer medulla. The mean number of TUNEL positively stained cells was calculated for quantification.

\section{Measurement of Oxidative} stress

Lipid peroxidation is an oxidative stress index that was assessed by quantifying the production of malondialdehyde (MDA) using the thiobarbituric acid reactive substance test (OxiSelect MDA Adduct ELISA Kit; Cell Biolabs Inc., San Diego, CA, USA). The MDA contents using whole kidney were detected according to the manufacturer's protocol.
Kidney Blood Press Res 2017;42:641-653

\begin{tabular}{l|l}
\hline DOI: $10.1159 / 000481804$ & (C) 2017 The Author(s). Published by S. Karger AG, Basel
\end{tabular} Published online: October 13, 2017 www.karger.com/kbr

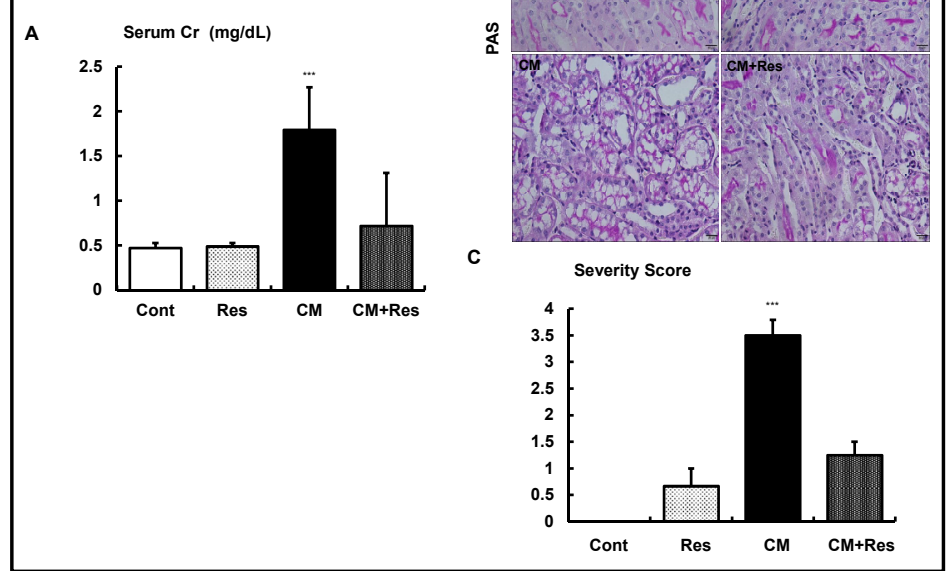

Fig. 1. Renal function and histological changes in iohexol- and resveratrol-treated mice kidneys. A: Effects of iohexol treatment and co-administration of resveratrol on serum creatinine levels in mice. B: Representative sections stained with periodic acid-Schiff (PAS) reagents in the outer medulla. Original magnification $\times 400$. Bar $=20$ $\mu \mathrm{m}$. C: Histological analysis of severity score for contrast-induced renal injury in the four experimental groups. Tubule vacuolization and loss of the brush border are scored for each group. ${ }^{* * *} \mathrm{p}<0.001 \mathrm{vs.} \mathrm{other} \mathrm{groups.}$ CIN, contrast-induced nephropathy; Cont, control; Res, resveratroltreated group; CM, contrast-media (iohexol)-treated group; CM + Res, contrast media plus resveratrol $(30 \mathrm{mg} / \mathrm{kg}$ intraperitoneally)-treated group.

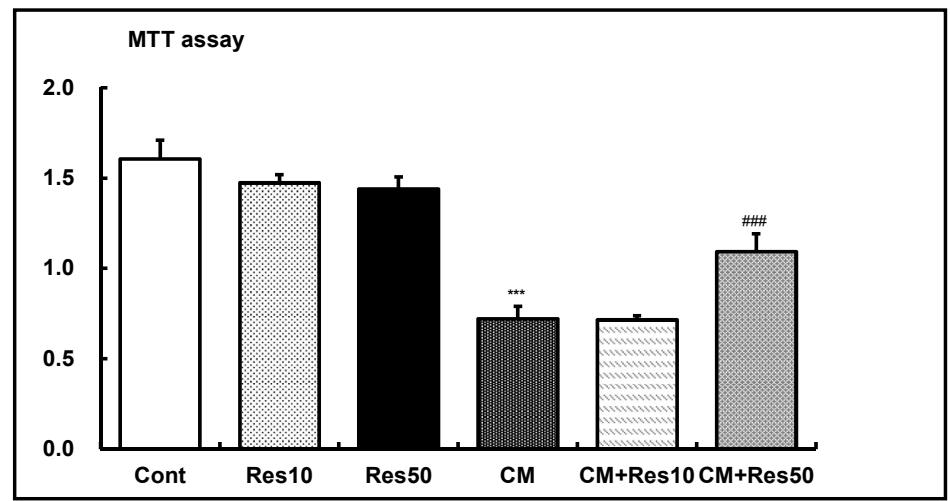

Fig. 2. Quantitative analysis of cell viability by 3-[4,5-dimethyl(thiazol2-yl)-3,5-dipheryl] tetradium bromide (MTT) assay in iohexol- and resveratrol-treated NRK-52E cells. Each column represents the mean \pm SE of three independent experiments. ${ }^{* * *} \mathrm{p}<0.001$ vs. other groups except the $\mathrm{CM}+\operatorname{Res} 10$ group, ${ }^{\# \# \#} \mathrm{p}<0.001$ vs. Cont and $\mathrm{CM}+\operatorname{Res} 10$ groups. 


\section{Kidney Blood Pressure Research}

Hong et al.: Renoprotective Effects of Resveratrol in CIN

\section{SIRT1 inhibition by SiRNA}

NRK-52E cells were transfected with small interfering (si) RNA, targeted to interfere with SIRT1 expression (Santa Cruz Biotechnology, CA, USA), or with siRNA control by complexing with transfection agents (G-Fectin; Genolution, Seoul, Korea), following the manufacturer's instructions. Approximately 24 hours after the transfection, iohexol or saline were added for 3 hours, and cell viability was measured using the 5-(3-carboxymethoxyphenyl)-2H-tetrazolium inner salt (MTS) cell proliferation assay (Promega Corp., Madison, WI, USA).

\section{Statistical analysis}

Data are expressed as means \pm standard error (SE). Differences between groups were examined for statistical significance by analysis of variance with the Bonferroni correction, using SPSS software, ver. 20.0 (IBM Corp. Armonk, NY, USA). A $p$ value $<0.05$ was considered significant.

\section{Results}

\section{Effects of resveratrol on renal functional and histological changes}

Serum creatinine levels in mice treated with iohexol increased significantly compared to those of saline-treated control mice (Cont vs. CM: $0.47 \pm 0.06$ vs. $1.79 \pm 0.48 \mathrm{mg} / \mathrm{dL}, p$

Fig. 3. Representative images and quantitative analysis of SIRT1 and PGC- $1 \alpha$ expression in vivo and in vitro after iohexol and resveratrol treatment. A: Representative immunoblot analysis of the effects of iohexol and resveratrol treatment on SIRT1 and PGC-1 $\alpha$ expression in mice kidneys $\mathrm{B}$ : Quantitative analyses for SIRT1/ $\beta$ actin. C: Quantitative analyses for PGC- $1 \alpha / \beta$-actin. D: Representative immunoblot analysis of the effects of iohexol and resveratrol on SIRT1 and PGC- $1 \alpha$ expression in rat proximal tubule cells. E: Quantitative analyses for SIRT1/ $\alpha$ tubulin. F: Quantitative analyses for PGC- $1 \alpha / \alpha$-tubulin. Each column represents the mean $\pm \mathrm{SE}$ of three independent experiments. ${ }^{*} \mathrm{p}<0.05,{ }^{* *} \mathrm{p}<0.01$ or ${ }^{* * *} \mathrm{p}<0.001$ vs. Cont group, ${ }^{\#} \mathrm{p}<0.05$ or ${ }^{\# \# \# ~} \mathrm{p}<0.001$ vs. Res group, ${ }^{\dagger} p<0.05,{ }^{+\dagger} p<0.01$ or ${ }^{++\dagger} \mathrm{p}<0.001$ vs. CM group. PGC- $1 \alpha$, peroxisome proliferator-activated receptor- $\gamma$ co-activator $1 \alpha$; Sirtuin 1, SIRT1; CIN, contrast-induced nephropathy; Cont, control; Res, resveratrol-treated group; CM, contrast media-treated group; $\mathrm{CM}+$ Res, contrast media plus resveratrol-treated group.

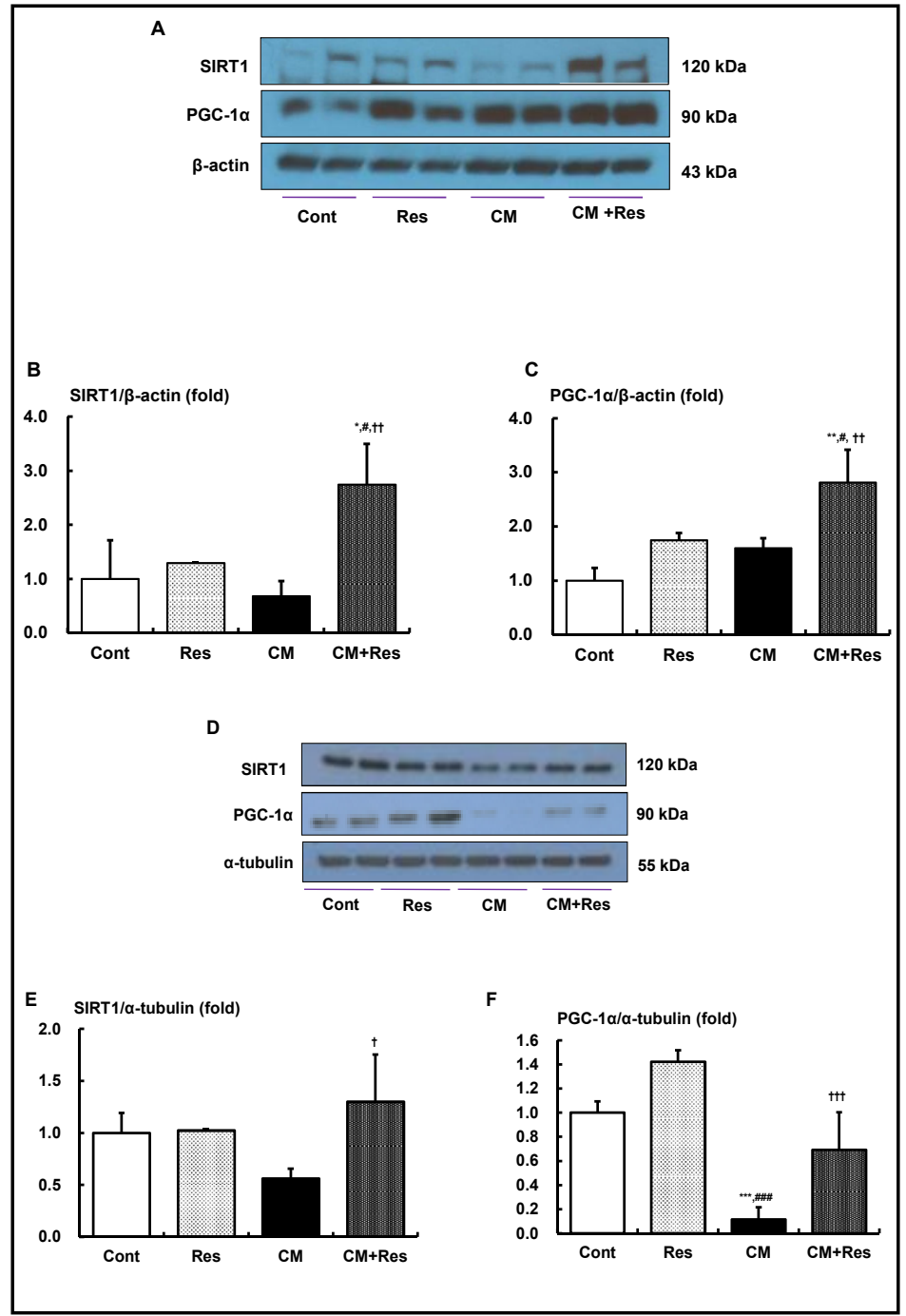




\section{Kidney Blood Pressure Research}

$<$ 0.001). Administering resveratrol with iohexol reduced serum creatinine level significantly compared to iohexol-only treated mice (CM vs. CM + Res: $1.79 \pm 0.48$ vs. $0.72 \pm 0.59$ mg/dL, Fig. 1A, $p<0.001$ ). The histological evaluation revealed significant tubular vacuolization and disruption of the tubular structures in the outer medulla after iohexol administration. Co-administration of resveratrol with iohexol significantly decreased the severity score for the tubular vacuolization and disruption of the tubular structures compared to the CM group in the outer medulla (Fig. 1B and C, $p<$ 0.001 ).

Effect of resveratrol on the toxicity of iohexol to NRK-52E cells

We assessed the effect of resveratrol on the cell viability of NRK-52E cells, renal proximal tubular cells of rat, after administration of iohexol. As shown in Fig. 2, pretreatment with resveratrol $(10$ or $50 \mu \mathrm{mol})$ had no significant effect on cell survival regardless of the concentration. Cell viability decreased significantly after iohexol treatment compared to the saline and resveratrol-only treatment groups (Cont vs. Res10 vs. Res50 vs. CM: 1.61 \pm 0.10 vs. $1.47 \pm 0.05$ vs. $1.44 \pm 0.07$ vs. $0.72 \pm 0.07$, $p<0.001$ ). Co-treatment of $50 \mu \mathrm{mol}$ resveratrol with iohexol effectively attenuated cell cytotoxicity (CM vs. CM + Res; $0.72 \pm 0.07$ vs. $1.09 \pm 0.01, p<0.001$ ).

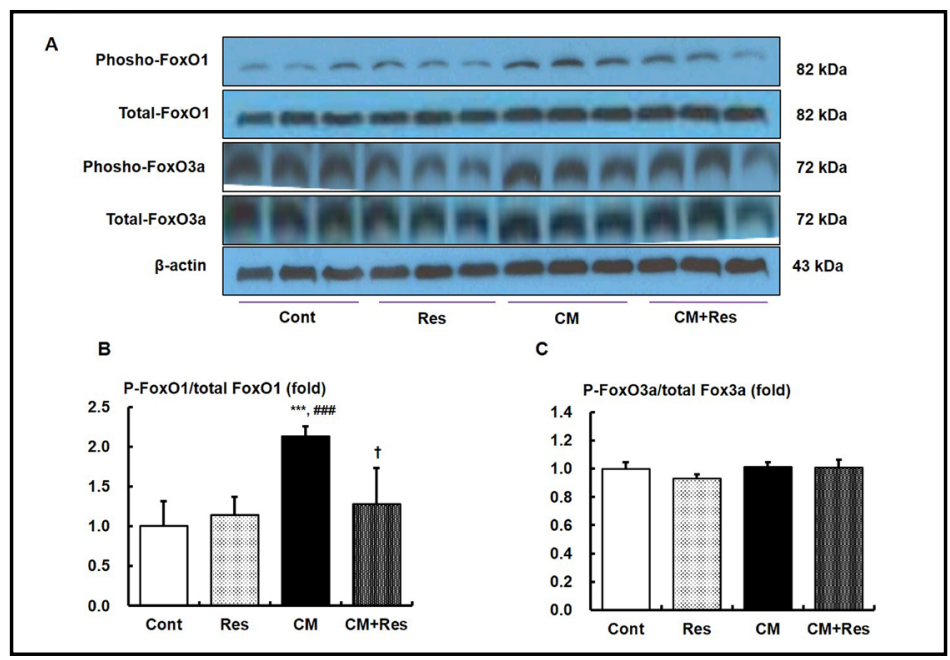

Fig. 4. Representative images and quantitative analysis of FoxOs expression in vivo after iohexol and resveratrol treatment. A: Representative immunoblot showing phospho-Ser ${ }^{253}$ FoxO3a, total Fox03a, phospho-Ser ${ }^{256}$ Fox01, and total Fox01 expression levels in mice kidneys. B: Quantitative analyses for phospho-Ser ${ }^{256}$ Fox01/ total Fox01. C: Quantitative analyses for phospho-Ser ${ }^{253}$ FoxO3a/total FoxO3a. Each column represents the mean \pm SE of three independent experiments. ${ }^{* * *} \mathrm{p}<0.001$ vs. Cont group, ${ }^{\# \# \# ~} \mathrm{p}<0.001$ vs. Res group, ${ }^{\dagger}$ $\mathrm{p}<0.05$ vs. CM group. FoxO, forkhead box 0; P-Fox01, phospho-Ser ${ }^{256}$; P-FoxO3a, phospho-Ser ${ }^{253}$ FoxO3a; CIN, contrast-induced nephropathy; Cont, control; Res, resveratrol-treated group; CM, contrast mediatreated group; $\mathrm{CM}+$ Res, contrast media plus resveratrol-treated group.

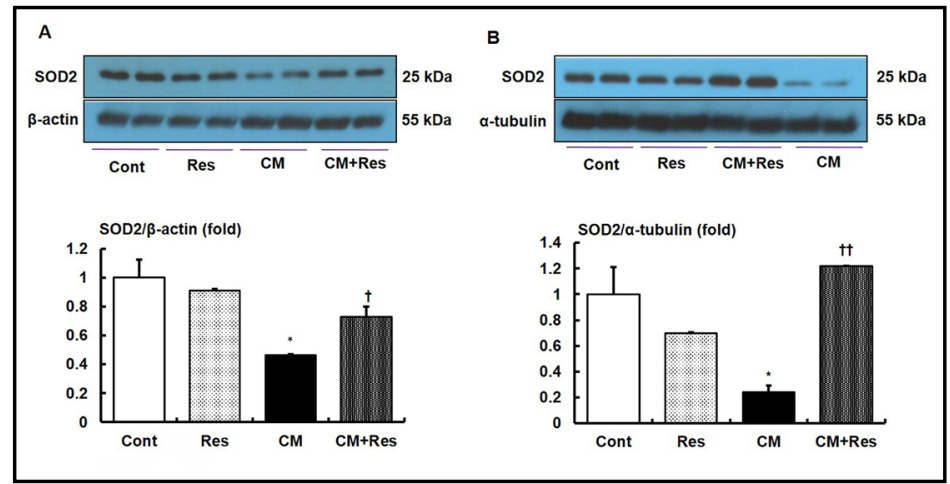

Fig. 5. Representative images and quantitative analysis of SOD2 expression in vivo and in vitro after iohexol and resveratrol treatment. A: Representative immunoblot and quantitative analysis of SOD2 in mice kidneys. B: Representative immunoblot and quantitative analysis of SOD2 in NRK-52E cells. Each column represents the mean \pm SE of three independent experiments. ${ }^{*} \mathrm{p}<0.05$ vs. Cont group, ${ }^{\dagger} \mathrm{p}<0.05$ or ${ }^{+\dagger} \mathrm{p}<0.01$ vs. CM group. SOD2, superoxide dismutase 2; Cont, control; Res, resveratrol-treated group; $\mathrm{CM}$, contrast media-treated group; CM + Res, contrast media plus resveratrol-treated group.
Kidney Blood Press Res 2017;42:641-653

\begin{tabular}{|l|l|}
\hline DOI: $10.1159 / 000481804$ & ( ) 2017 The Author(s). Published by S. Karger AG, Basel \\
\hline
\end{tabular} Published online: October 13, 2017 www.karger.com/kbr 


\section{Kidney Blood Pressure Research}

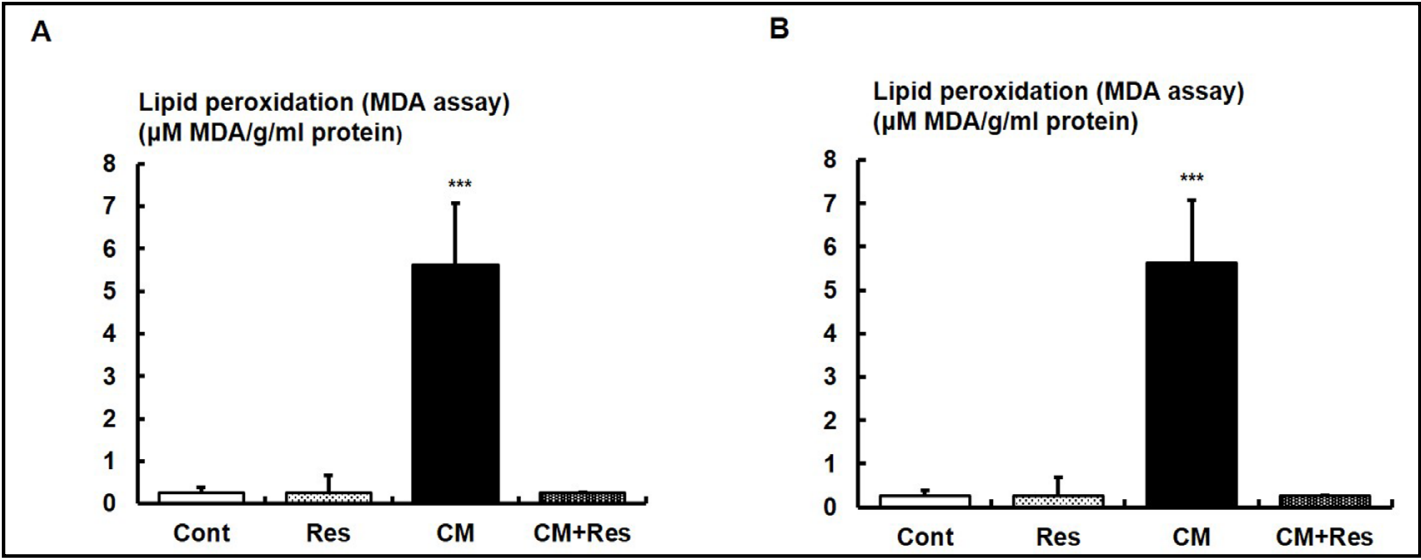

Fig. 6. Concentrations of the lipid peroxidation marker malondialdehyde (MDA) in (A) whole kidney tissues of mice and (B) NRK-52E cells. ${ }^{* * *} \mathrm{p}<0.001$ vs. other groups. Cont, control; Res, resveratrol-treated group; CM, contrast media-treated group; $\mathrm{CM}+$ Res, contrast media plus resveratrol-treated group.

Fig. 7. Representative images and quantitative TUNEL and cleaved caspase-3 analyses in vivo and in vitro after iohexol and resveratrol treatment. A: Representative immunohistochemical staining and quantitative analysis for TUNELpositive cells in the outer medulla of the experimental groups. Each column represents the mean \pm SEM of three independent experiments. Original magnification $\times 400$. Bar $=$ $20 \mu \mathrm{m} .{ }^{* * *} \mathrm{p}<0.001$ vs. other groups. B: Representative immunoblot and quantitative analysis of the cleaved caspase-3 results in mice kidneys. ${ }^{* *} \quad \mathrm{p}<0.01$ vs. other groups. C: Representative immunoblot and quantitative analysis of the cleaved caspase-3 results in NRK-52E

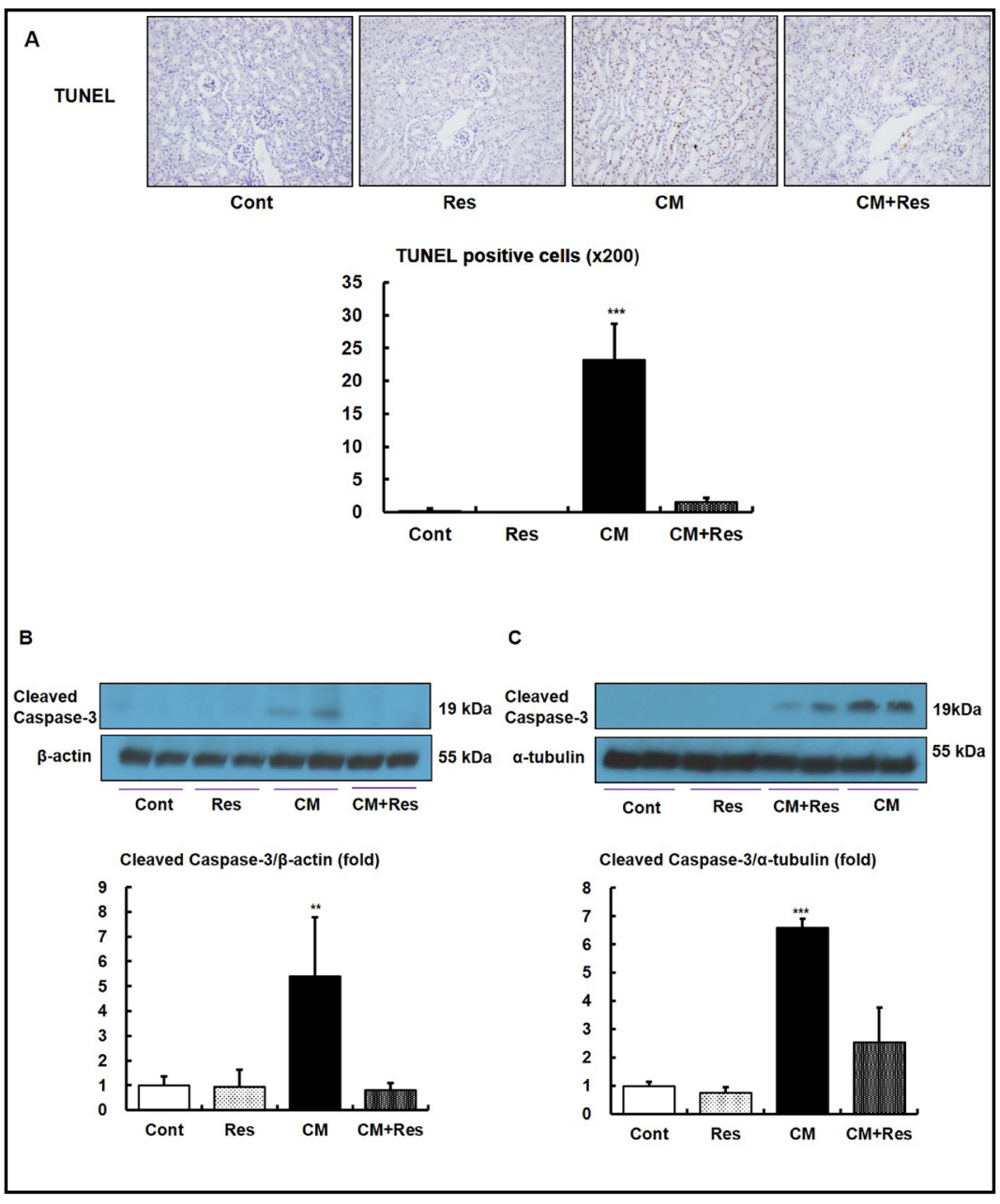
cells. Each column represents the mean \pm SE of three independent experiments. ${ }^{* *} p<0.001$ vs. other groups. TUNEL, terminal deoxynucleotidyl transferase dUTP nick end labeling; Cont, control; Res, resveratrol-treated group; CM, contrast media-treated group; $\mathrm{CM}+$ Res, contrast media plus resveratrol-treated group. 


\section{Kidney Blood Pressure Research}

\section{Effects \\ resveratrol \\ SIRT1 and PGC-1 $\alpha$ expression levels in kidney tissues and tubular cells}

To investigate the molecular mechanisms associating the renoprotective effects of resveratrol in mice with CIN, we measured intra-renal SIRT1 and PGC-1 $\alpha$ expression levels in mice kidneys using an immunoblot analysis. Administering iohexol decreased SIRT1 expression in mice kidneys compared to the saline and resveratrol-only treatment groups, whereas co-administration of resveratrol with iohexol effectively upregulated renal SIRT1 expression (Fig. 3A and $\mathrm{B}, p<0.01$ ). PGC- $1 \alpha$ expression in the kidneys was increased mildly in the resveratrol-only treatment and iohexolonly treatment groups compared to the saline-control group, but the differences between groups were not significant. Co-administering resveratrol with iohexol significantly accentuated PGC$1 \alpha$ expression in mice kidneys (Fig. 3A and C, $p<0.01$ ). The expression of SIRT1 and PGC- $1 \alpha$ were also examined in NRK$52 \mathrm{E}$ cells. Iohexol treatment significantly decreased the expressions of SIRT1 and PGC- $1 \alpha$ compared to those in saline and resveratrol-only treated cells, whereas co-treatment with resveratrol and iohexol effectively restored SIRT1 and PGC- $1 \alpha$ expression (Fig. 3D-F, $p<0.05$ and $p<$ 0.001 , respectively)

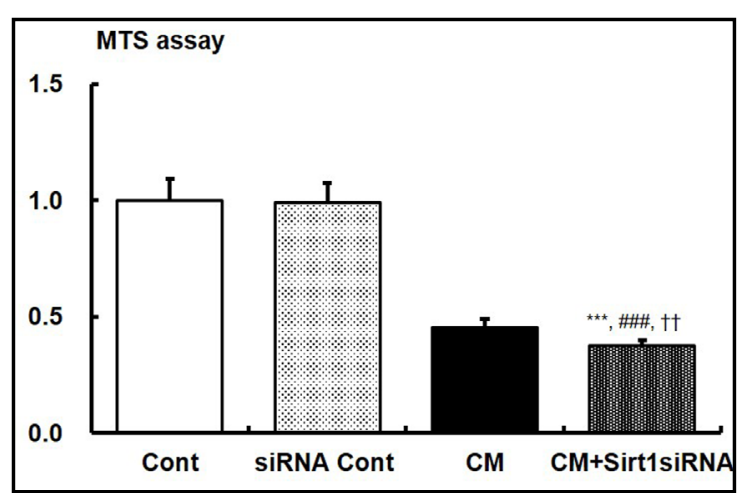

Fig. 9. Quantitative analysis of cell viability by the 5-(3-carboxymethoxyphenyl)-2H-tetrazolium inner salt (MTS) assay after iohexol treatment and inhibited SIRT1 expression after siRNA transfection in NRK$52 \mathrm{E}$ cells. Each column represents the mean \pm SE of three independent experiments. ${ }^{* * *} \mathrm{p}<0.001$ vs. Cont group, ${ }^{\# \#} \mathrm{p}<0.001$ vs. siRNA Cont group, ${ }^{\dagger+} \mathrm{p}<0.01$ vs. CM groups. SIRT1, sirtuin 1; siRNA, small interfering RNA; Cont, control; Res, resveratrol-treated group; $\mathrm{CM}$, contrast media-treated group; $\mathrm{CM}+$ Res, contrast media plus resveratrol-treated group.

\section{Effects of resveratrol on renal expression of FoxOs and SOD2}

Phospho-Ser ${ }^{256}$ FoxO1 increased significantly in mice kidneys after administration of iohexol compared to the saline and resveratrol-only treatment groups. Co-administering resveratrol with iohexol markedly decreased phospho-Ser ${ }^{256}$ FoxO1 expression (Fig. 4A and $\mathrm{B}, p<0.05)$. However, the expression of phospho-Ser ${ }^{253}$ FoxO3a did not change significantly after administering iohexol or resveratrol (Fig. 4A and C). Expression of SOD2, which is an antioxidant and mitochondrial form of SOD, decreased significantly in the iohexol-only 


\section{Kidney Blood Pressure Research}

treated group compared with the resveratrol-only treated groups in vivo and in vitro. Cotreatment of resveratrol with iohexol significantly restored SOD2 expression in mice kidneys and NRK-52E cells (Fig. 5A and B, $p<0.05$ and $p<0.01$, respectively).

\section{Effects of resveratrol on lipid peroxidation}

Oxidative stress measured by the MDA production assay showed that iohexol increased the MDA level significantly in vivo and in vitro compared to the saline and resveratrol-only treatment groups, and co-treatment of resveratrol with iohexol significantly alleviated oxidative stress (Fig. $6 \mathrm{~A}$ and $\mathrm{B}, p<0.001$ ).

\section{Effects of resveratrol on renal apoptosis}

The degree of tubular apoptosis in kidneys was assessed by TUNEL staining and increased significantly after administering iohexol compared to the saline and resveratrolonly treatment mice. Co-administering resveratrol and iohexol significantly reduced the number of TUNEL-positive cells, by approximately 95\% in the outer medulla (Fig. 7A, $p<$ 0.001 ). The expression of cleaved caspase- 3 also increased significantly in the iohexol-only treated mice kidneys compared to the saline and resveratrol-only treatment groups, and coadministration of resveratrol with iohexol markedly decreased cleaved caspase-3 protein expression level (Fig. 7B, $p<0.01$ ). These results were shown consistently in an in vitro study, where cleaved caspase-3 increased in iohexol-only treated cells compared to the saline and resveratrol-only treated groups and decreased after co-treatment of resveratrol with iohexol (Fig. 7C, $p<0.001$ ).

\section{Effect of resveratrol on macrophage infiltration}

Only a few macrophages were detected in the kidneys of the saline and resveratrol-only treated mice. Macrophage infiltration into kidneys, as measured by F4/80 staining, increased significantly after administering iohexol, and decreased significantly by co-administrating resveratrol and iohexol in the outer medulla (Fig. 8, $p<0.001$ ).

Downregulation of SIRT1 enhances the decrease in NRK-52E cell viability caused by iohexol

SIRT1 expression in renal tubular cells was significantly downregulated by SIRT1 siRNA treatment (data not shown). Cell viability and proliferation, as analyzed by the MTS assay, remained unchanged after transfecting siRNA silencing SIRT1 (siRNA Cont group) compared to the negative siRNA-treated group (Cont group). The iohexol treatment significantly reduced cell viability and proliferation compared to the Cont and siRNA Cont groups, and SIRT1 siRNA-treated renal tubular cells with iohexol accentuated the decrease in cell viability caused by iohexol (CM vs. CM + Sirt1 siRNA; $0.45 \pm 0.04$ vs. $0.37 \pm 0.02$, Fig. $9, p<0.01$ ).

\section{Discussion}

This study demonstrated that renal dysfunction in mice with CIN was accompanied by increased oxidative stress and renal apoptosis and decreased SIRT1 expression in the kidneys. CIN suppressed the SIRT1-PGC-1 $\alpha$ signaling pathway, followed by inactivation of Fox01 and a decrease in mitochondrial SOD2. The recovery of SIRT1 and PGC- $1 \alpha$ expression by resveratrol was associated with a renoprotective effect in CIN through the attenuation of apoptosis and oxidative stress in vivo and in vitro.

Oxidative stress has been proposed to be an important mechanism in the pathogenesis of CIN [8]. Iodinated CM decreases renal medullary oxygenation, which leads to increased production of ROS or its metabolites in the kidneys [24,25]. ROS scavengers, including various antioxidants such as $\mathrm{N}$-acetylcysteine, sodium bicarbonate, $\beta$-blocker and theophylline, are helpful for preventing CIN [26-28]. Although some studies demonstrated the efficacy of these preventive measures, the role of ROS scavengers in CIN has not been confirmed because of 


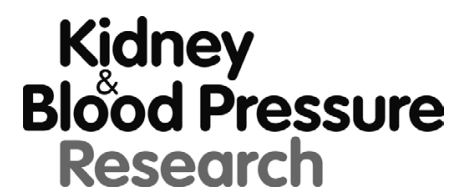

\begin{tabular}{l|l}
\hline Kidney Blood Press Res 2017;42:641-653 \\
\hline DOI: 10.1159/000481804 & $\begin{array}{l}\text { (c) 2017 The Author(s). Published by S. Karger AG, Basel } \\
\text { www.karger.com/kbr }\end{array}$ \\
\hline Published online: October 13, 2017
\end{tabular}

Published onlne:October 13,2017 www.karger.com/kbr

inconsistencies between studies. There is a need to reveal the exact mechanisms of CIN and trials with new therapeutic and preventive approaches based on the mechanisms have been approved.

Resveratrol (3, 5,4'-trihydroxystilbene) is a natural polyphenolic phytoalexin in many plants, such as red wine, berries, grapes and peanut skins [29]. Resveratrol exerts its cytoprotective effects by regulating antioxidant activity and activating SIRT1 expression. Resveratrol increases SIRT1 activity through an allosteric interaction, resulting in increased affinity of SIRT1 for NAD ${ }^{+}$and an acetylated substrate to scavenge ROS directly and modulate the expression and activity of other antioxidant enzymes [30, 31]. An increase in SIRT1 expression in the kidneys ameliorates cisplatin-induced oxidative stress and tubular cell damage in renal tubule-specific Sirt1 transgenic mice [27]. Ischemia reperfusion-induced acute kidney injury decreases after the activation of SIRT1, but aggravates in SIRT1 knockout mice [32]. The present study revealed that reduced SIRT1 expression was substantially associated with CM-induced renal damage in vivo and in vitro, and that pharmacological activation of SIRT1 by resveratrol contributed to a renoprotective effect in mice with CIN. In addition, inhibition of SIRT1 expression significantly accentuated the cytotoxicity by CM in cultured renal tubular cells. Taken together, these observations suggest that renal SIRT1 is a potential therapeutic target for CIN.

Our study also demonstrated that PGC- $1 \alpha$ is a possible mediator of the effect of SIRT1 on CIN. PGC- $1 \alpha$ is a master transcriptional coactivator that facilitates combustion of stored energy by increasing mitochondrial production and subsequently influences essential biologic processes such as aging and oxidative stress [33]. Various experimental models of acute kidney injury have been demonstrated the renoprotective effects through the activation of PGC- $1 \alpha$. Tran et al. showed that PGC- $1 \alpha$ plays an important role in recovery of endotoxininduced acute kidney injury through regulation of mitochondrial biogenesis [34]. Another study also reported that deacetylation of PGC- $1 \alpha$ by activating SIRT1 expression ameliorates renal ischemic-reperfusion injury by recovering mitochondrial homeostasis [16]. This is the first study to elucidate a renoprotective role for SIRT1-PGC-1 $\alpha$ signaling after resveratrol treatment in a CIN model.

FoxOs have been known to function together with PGC- $1 \alpha$ and are key transcription factors that control the balance of various biological processes, including glucose metabolism, apoptosis and detoxification of ROS [35]. The reciprocal relationship between FoxOs and PGC$1 \alpha$ activation is associated with regulation of oxidative stress [36]. One study demonstrated that nuclear FoxO1 protein expression, as a downstream of the p38 MAPK pathway, increases in CIN and can be blocked by Chinese herbal medication [37]. We further evaluated the FoxO transcription factors signaling pathway, which is associated with oxidative stress regulated by SIRT1-PGC-1a. We initially hypothesized that both Fox01 and FoxO3a would be inactivated in mice with CIN. However, Fox01 was inactivated, as shown by increased phosphorylation of FoxO1, whereas FoxO3a was no change in mice with CIN Resveratrol pretreatment was significantly dephosphorylation of FoxO1, which was associated with a decrease of oxidative stress, particularly SOD2, which attenuated CIN. These findings suggest that resveratrol variously regulates different subtypes of FoxOs in CIN.

The attenuation of CIN by resveratrol was accompanied by reduced renal tubular cell apoptosis. Apoptosis is one of the pathological mechanisms of CIN [38]. In this study, we found that caspase-3 activation was induced by iohexol treatment, which resulted in increased the number of TUNEL-positive cells in outer medulla, and resveratrol treatment effectively reduced the apoptosis induced by iohexol. Resveratrol leads to decrease tubular apoptosis by activating SIRT1-PGC- $1 \alpha$ signaling and its downstream FoxO pathway $[39,40]$. The increase in oxidative stress and apoptosis in response to iohexol-induced infiltration of macrophages into injured tissues decreased with co-administration of resveratrol.

Renal medullary hypoxia plays an important role for the pathophysiology of CIN $[41,42]$. CM-induced medullary hypoxia may increase the formation of ROS, and hypoxia-inducible factor (HIF)-1 $\alpha$, the master switch of hypoxic adaptation responses, was induced in the outer 


\section{Kidney \\ Blood Pressure Research}

and inner medulla in a rat model after the administration of CM [43]. In spite of conflict results for the association between SIRT1 and HIF-1 $\alpha$ expression, recent previous study demonstrated that SIRT1 led to activation of HIF- $1 \alpha$ in cancer cell lines, which is a positive regulator of HIF- $1 \alpha$ transcriptional activity in response to hypoxia [44]. In addition, PGC-1 $\alpha$ activation inhibited hydroxylation of the oxygen-dependent degradation domain of HIF- $1 \alpha$, and led to HIF-1 $\alpha$ stabilization by decreasing intracellular ubiquitination and degradation in skeletal muscle cells. Subsequently, PGC- $1 \alpha$-induced HIF- $1 \alpha$ activation facilitated an increase in oxygen supply and attenuated ROS production [45]. To date, the association among SIRT1, PGC- $1 \alpha$ and HIF- $1 \alpha$ expression did not confirm in the tubular cells under hypoxia. Therefore, it should be studied further about the detailed mechanisms of resveratrol effect regarding hypoxia caused by CM.

\section{Conclusion}

This study demonstrated that activating SIRT1-PGC-1 $\alpha$-FoxO1 signaling mediated the renoprotective effect of resveratrol by decreasing oxidative stress and apoptosis. SIRT1 provides a potential therapeutic target to minimize CIN.

\section{Disclosure Statement}

The authors have no financial and commercial conflicts of interest.

\section{Acknowledgements}

This research was supported by Clinical Research Institute Grant (01400021) funded by Korea University Guro Hospital.

\section{References}

1 Rudnick MR, Goldfarb S, Tumlin J: Contrast-induced nephropathy: is the picture any clearer? Clin J Am Soc Nephrol 2008;3:261-262.

-2 Tepel M, Aspelin P, Lameire N: Contrast-induced nephropathy: a clinical and evidence-based approach. Circulation 2006;113:1799-1806.

3 Barrett BJ, Parfrey PS: Clinical practice. Preventing nephropathy induced by contrast medium. N Engl J Med 2006;354:379-386.

4 Stacul F, Adam A, Becker CR, Davidson C, Lameire N, McCullough PA, Tumlin J: Strategies to reduce the risk of contrast-induced nephropathy. Am J Cardiol 2006;98:59K-77K.

5 Burchardt P, Rzezniczak J, Synowiec T, Angerer D, Palasz A, Zurawski J: Lack of Serum Creatinine Decrease After Coronary Angiography Despite Prophylactic Hydration After Routine Coronary Angiography/ Angioplasty in Stable Angina Patients--Pilot Study. Kidney Blood Press Res 2016;41:222-230.

6 Tumlin J, Stacul F, Adam A, Becker CR, Davidson C, Lameire N, McCullough PA: Pathophysiology of contrastinduced nephropathy. Am J Cardiol 2006;98:14K-20K.

-7 Hizoh I, Haller C: Radiocontrast-induced renal tubular cell apoptosis: hypertonic versus oxidative stress. Invest Radiol 2002;37:428-434.

8 Michael A, Faga T, Pisani A, Riccio E, Bramanti P, Sabbatini M, Navarra M, Andreucci M: Molecular mechanisms of renal cellular nephrotoxicity due to radiocontrast media. Biomed Res Int 2014;2014:249810.

-9 Afshar G, Murnane JP: Characterization of a human gene with sequence homology to Saccharomyces cerevisiae SIR2. Gene 1999;234:161-168. 


\section{Kidney \\ Blood Pressure Research}

Hong et al.: Renoprotective Effects of Resveratrol in CIN

10 Vaziri H, Dessain SK, Ng Eaton E, Imai SI, Frye RA, Pandita TK, Guarente L, Weinberg RA: hSIR2(SIRT1) functions as an NAD-dependent p53 deacetylase. Cell 2001;107:149-159.

11 Brunet A, Sweeney LB, Sturgill JF, Chua KF, Greer PL, Lin Y, Tran H, Ross SE, Mostoslavsky R, Cohen HY, Hu LS, Cheng HL, Jedrychowski MP, Gygi SP, Sinclair DA, Alt FW, Greenberg ME: Stress-dependent regulation of FOXO transcription factors by the SIRT1 deacetylase. Science 2004;303:2011-2015.

12 Rodgers JT, Lerin C, Haas W, Gygi SP, Spiegelman BM, Puigserver P: Nutrient control of glucose homeostasis through a complex of PGC-1alpha and SIRT1. Nature 2005;434:113-118.

$\checkmark 13$ Yeung F, Hoberg JE, Ramsey CS, Keller MD, Jones DR, Frye RA, Mayo MW: Modulation of NF-kappaBdependent transcription and cell survival by the SIRT1 deacetylase. EMBO J 2004;23:2369-2380.

14 He W, Wang Y, Zhang MZ, You L, Davis LS, Fan H, Yang HC, Fogo AB, Zent R, Harris RC, Breyer MD, Hao CM: Sirt1 activation protects the mouse renal medulla from oxidative injury. J Clin Invest 2010;120:1056-1068.

15 Kim DH, Jung YJ, Lee JE, Lee AS, Kang KP, Lee S, Park SK, Han MK, Lee SY, Ramkumar KM, Sung MJ, Kim W: SIRT1 activation by resveratrol ameliorates cisplatin-induced renal injury through deacetylation of p53. Am J Physiol Renal Physiol 2011;301:F427-435.

16 Funk JA, Schnellmann RG: Accelerated recovery of renal mitochondrial and tubule homeostasis with SIRT1/PGC-1alpha activation following ischemia-reperfusion injury. Toxicol Appl Pharmacol 2013;273:345-354.

17 Gu J, Yang M, Qi N, Mei S, Chen J, Song S, Jing Y, Chen M, He L, Sun L, Hu H, Li L, Wuthrich RP, Wu M, Mei C: Olmesartan Prevents Microalbuminuria in db/db Diabetic Mice Through Inhibition of Angiotensin II/p38/ SIRT1-Induced Podocyte Apoptosis. Kidney Blood Press Res 2016;41:848-864.

18 Valle I, Alvarez-Barrientos A, Arza E, Lamas S, Monsalve M: PGC-1alpha regulates the mitochondrial antioxidant defense system in vascular endothelial cells. Cardiovasc Res 2005;66:562-573.

19 St-Pierre J, Drori S, Uldry M, Silvaggi JM, Rhee J, Jager S, Handschin C, Zheng K, Lin J, Yang W, Simon DK, Bachoo R, Spiegelman BM: Suppression of reactive oxygen species and neurodegeneration by the PGC-1 transcriptional coactivators. Cell 2006;127:397-408.

20 Kitada M, Koya D: Renal Protective Effects of Resveratrol. Oxid Med Cell Longev 2013;2013:568093.

21 Lee HT, Jan M, Bae SC, Joo JD, Goubaeva FR, Yang J, Kim M: A1 adenosine receptor knockout mice are protected against acute radiocontrast nephropathy in vivo. Am J Physiol Renal Physiol 2006;290:F13671375.

22 Jablonski P, Howden BO, Rae DA, Birrell CS, Marshall VC, Tange J: An experimental model for assessment of renal recovery from warm ischemia. Transplantation 1983;35:198-204.

-23 Billings FT, Chen SW, Kim M, Park SW, Song JH, Wang S, Herman J, D’Agati V, Lee HT: alpha2-Adrenergic agonists protect against radiocontrast-induced nephropathy in mice. Am J Physiol Renal Physiol 2008;295:F741-748.

24 Kongkham S, Sriwong S, Tasanarong A: Protective effect of alpha tocopherol on contrast-induced nephropathy in rats. Nefrologia 2013;33:116-123.

-25 Toprak 0, Cirit M, Tanrisev M, Yazici C, Canoz O, Sipahioglu M, Uzum A, Ersoy R, Sozmen EY: Preventive effect of nebivolol on contrast-induced nephropathy in rats. Nephrol Dial Transplant 2008;23:853-859.

-26 Briguori C, Quintavalle C, De Micco F, Condorelli G: Nephrotoxicity of contrast media and protective effects of acetylcysteine. Arch Toxicol 2011;85:165-173.

27 Efrati S, Berman S, Ilgiyeav I, Siman-Tov Y, Averbukh Z, Weissgarten J: Differential effects of $\mathrm{N}$-acetylcysteine, theophylline or bicarbonate on contrast-induced rat renal vasoconstriction. Am J Nephrol 2009;29:181-191.

-28 Altunoren O, Balli M, Eren N, Tasolar H, Arpaci A, Caglayan CE, Yavuz YC, Gungor O: Is Nebivolol Really Effective in Preventing Contrast Induced Nephropathy? Kidney Blood Press Res 2015;40:533-541.

29 Bertelli AA, Das DK: Grapes, wines, resveratrol, and heart health. J Cardiovasc Pharmacol 2009;54:468-476.

-30 Howitz KT, Bitterman KJ, Cohen HY, Lamming DW, Lavu S, Wood JG, Zipkin RE, Chung P, Kisielewski A, Zhang LL, Scherer B, Sinclair DA: Small molecule activators of sirtuins extend Saccharomyces cerevisiae lifespan. Nature 2003;425:191-196.

31 Leonard SS, Xia C, Jiang BH, Stinefelt B, Klandorf H, Harris GK, Shi X: Resveratrol scavenges reactive oxygen species and effects radical-induced cellular responses. Biochem Biophys Res Commun 2003;309:10171026. 


\section{Kidney \\ Blood Pressure Research}

-32 Fan H, Yang HC, You L, Wang YY, He WJ, Hao CM: The histone deacetylase, SIRT1, contributes to the resistance of young mice to ischemia/reperfusion-induced acute kidney injury. Kidney Int 2013;83:404413.

-33 Puigserver P, Rhee J, Donovan J, Walkey CJ, Yoon JC, Oriente F, Kitamura Y, Altomonte J, Dong H, Accili D, Spiegelman BM: Insulin-regulated hepatic gluconeogenesis through FOX01-PGC-1alpha interaction. Nature 2003;423:550-555.

-34 Tran M, Tam D, Bardia A, Bhasin M, Rowe GC, Kher A, Zsengeller ZK, Akhavan-Sharif MR, Khankin EV, Saintgeniez M, David S, Burstein D, Karumanchi SA, Stillman IE, Arany Z, Parikh SM: PGC-1alpha promotes recovery after acute kidney injury during systemic inflammation in mice. J Clin Invest 2011;121:40034014.

35 Van Der Heide LP, Hoekman MF, Smidt MP: The ins and outs of FoxO shuttling: mechanisms of FoxO translocation and transcriptional regulation. Biochem J 2004;380:297-309.

36 Daitoku H, Yamagata K, Matsuzaki H, Hatta M, Fukamizu A: Regulation of PGC-1 promoter activity by protein kinase B and the forkhead transcription factor FKHR. Diabetes 2003;52:642-649.

-37 Gong X, Wang Q Tang X, Wang Y, Fu D, Lu H, Wang G, Norgren S: Tetramethylpyrazine prevents contrastinduced nephropathy by inhibiting p38 MAPK and FoxO1 signaling pathways. Am J Nephrol 2013;37:199207.

-38 Yano T, Itoh Y, Sendo T, Kubota T, Oishi R: Cyclic AMP reverses radiocontrast media-induced apoptosis in LLC-PK1 cells by activating A kinase/PI3 kinase. Kidney Int 2003;64:2052-2063.

-39 Hasegawa K, Wakino S, Yoshioka K, Tatematsu S, Hara Y, Minakuchi H, Sueyasu K, Washida N, Tokuyama H, Tzukerman M, Skorecki K, Hayashi K, Itoh H: Kidney-specific overexpression of Sirt1 protects against acute kidney injury by retaining peroxisome function. J Biol Chem 2010;285:13045-13056.

-40 Sin TK, Yu AP, Yung BY, Yip SP, Chan LW, Wong CS, Ying M, Rudd JA, Siu PM: Modulating effect of SIRT1 activation induced by resveratrol on Foxo1-associated apoptotic signalling in senescent heart. J Physiol 2014

41 Heyman SN, Rosen S, Rosenberger C: Renal parenchymal hypoxia, hypoxia adaptation, and the pathogenesis of radiocontrast nephropathy. Clin J Am Soc Nephrol 2008;3:288-296.

42 Persson PB, Hansell P, Liss P: Pathophysiology of contrast medium-induced nephropathy. Kidney Int 2005;68:14-22.

43 Rosenberger C, Heyman SN, Rosen S, Shina A, Goldfarb M, Griethe W, Frei U, Reinke P, Bachmann S, Eckardt $\mathrm{KU}$ : Up-regulation of HIF in experimental acute renal failure: evidence for a protective transcriptional response to hypoxia. Kidney Int 2005;67:531-542.

44 Laemmle A, Lechleiter A, Roh V, Schwarz C, Portmann S, Furer C, Keogh A, Tschan MP, Candinas D, Vorburger SA, Stroka D: Inhibition of SIRT1 impairs the accumulation and transcriptional activity of HIF1alpha protein under hypoxic conditions. PLoS One 2012;7:e33433.

-45 O'Hagan KA, Cocchiglia S, Zhdanov AV, Tambuwala MM, Cummins EP, Monfared M, Agbor TA, Garvey JF, Papkovsky DB, Taylor CT, Allan BB: PGC-1alpha is coupled to HIF-1alpha-dependent gene expression by increasing mitochondrial oxygen consumption in skeletal muscle cells. Proc Natl Acad Sci U S A 2009;106:2188-2193. 